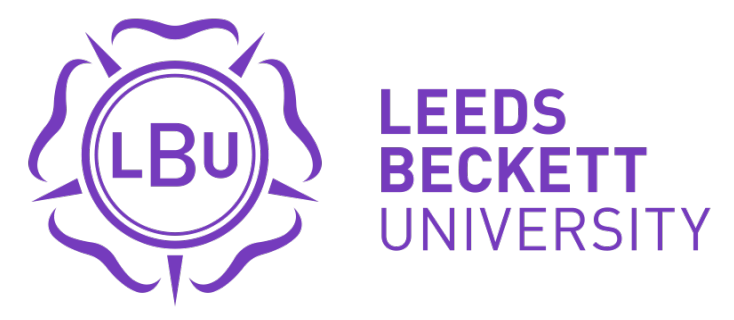

Citation:

Morley, D and Pyke, D and Till, KA (2015) An investigation into the use of a movement assessment protocol for under 14 rugby league players in a talent development environment. International Journal of Sports Science and Coaching, 10 (4). ISSN 1747-9541 DOI: https://doi.org/10.1260/17479541.10.4.623

Link to Leeds Beckett Repository record:

https://eprints.leedsbeckett.ac.uk/id/eprint/2152/

Document Version:

Article (Published Version)

The aim of the Leeds Beckett Repository is to provide open access to our research, as required by funder policies and permitted by publishers and copyright law.

The Leeds Beckett repository holds a wide range of publications, each of which has been checked for copyright and the relevant embargo period has been applied by the Research Services team.

We operate on a standard take-down policy. If you are the author or publisher of an output and you would like it removed from the repository, please contact us and we will investigate on a case-by-case basis.

Each thesis in the repository has been cleared where necessary by the author for third party copyright. If you would like a thesis to be removed from the repository or believe there is an issue with copyright, please contact us on openaccess@leedsbeckett.ac.uk and we will investigate on a case-by-case basis. 


\title{
An Investigation into the Use of a Movement Assessment Protocol for Under-14 Rugby League Players in a Talent Development Environment
}

\author{
David Morley', Daniel Pyke ${ }^{2}$ and Kevin Till2 \\ ${ }^{1}$ School of Education Leisure and Sport Studies, IM Marsh Campus, \\ Barkhill Road, Liverpool, L17 6BD, UK \\ E-mail: D.M.Morley@ljmu.ac.uk \\ 2Leeds Beckett University, Carnegie Faculty of Sport \& Education \\ Fairfax Hall, Headingley Campus, Leeds, LS6 3QS, UK
}

\begin{abstract}
This study investigated the use of a movement assessment protocol for under-14 rugby league players by evaluating the relationships between chronological age, maturation, and anthropometry, and fitness and qualitative movement assessments (QMA) of 84 rugby league players within a talent development environment. A one-way ANOVA showed Quartile 1 players were more mature, taller $(173.0 \pm 7.4 \mathrm{vs} 165.0 \pm 8.0 \mathrm{~cm}$ ) and heavier (72.5 vs $58.7 \mathrm{~kg}$ ) than Quartile 4 players, with no difference evident for fitness or QMA measures. Earlier maturing players had significantly greater upper body power $(5.39 \pm 0.46$ vs $4.42 \pm 0.68 \mathrm{~m}), 20 \mathrm{~m}$ speed (3.48 \pm 0.14 vs 3.65 $\pm 0.19 s)$ and power pass QMA (13.88 \pm 2.18 vs $12.00 \pm 1.98)$ than later maturing players. Body mass was positively related to power pass fitness ( $r=0.50)$ and QMA ( $r=0.22)$ scores, with negative relationships found for vertical jump performance $(r=-0.24)$, sprint QMA $(r=-.29)$ and turn off either foot QMA ( $r=-0.26)$. There is a need to educate coaches about the use of both fitness testing and qualitative movement assessments to identify talented U14 rugby league players, which potentially reduces relative age and maturational biases.
\end{abstract}

Key words: Anthropometry, Fitness Testing, Maturation, Qualitative Movement Assessment, Relative Age Effect, Rugby Football

\section{INTRODUCTION}

The Rugby Football League (RFL) is the governing body for Rugby League football in the UK, with almost 250,000 people currently involved as players, coaches, match officials or volunteers and 90,000 children taking part in the Champion schools knock-out competition each year [1]. The development of young players is of great importance to the game and it is

Reviewers: John Cronin (Auckland University of Technology, New Zealand)

Tim Gabbett (Australia Catholic University, Australia)

Rudi Meir (Southern Cross University, Australia) 
believed that by facilitating a smooth transition at the adolescent stage, the quality of players progressing from junior to senior level can be increased [2]. In alignment with the recognition of the necessity to ensure a 'smooth transition', the RFL piloted a talent development intervention, named Embed the Pathway (EtP) within the Under 14 age category. A purpose of EtP was to assess player 'movement' and 'physical' capabilities within a 6-panel talent development framework used by the National Governing Body [3]. The assessment of the Under 14 age group was of particular interest to the RFL as this was a key period of maturation for the players in terms of transiting through Peak Height Velocity [4] and players were about to be formally selected onto the first exclusive segment of the RFL talent pathway.

This study explored the relationship between the results of fitness and movement assessments, relative age, maturation and anthropometric characteristics of Under 14 junior Rugby League players, within the context of a talent development intervention. A 'dualistic' approach was selected for the Under 14 players that relied upon a quantitative approach, drawing from fitness assessments, traditionally associated with talent identification practices in rugby league across junior and senior levels [5,6], and a qualitative approach that aimed to assess movement competency by analysing the movement process rather than performance outcome [7].

By combining these two approaches, and assessing any relationships with pre-determined developmental characteristics, the study aimed to inform the development of a subsequent assessment protocol to be used on the wholesale delivery of EtP, nationally. The majority of assessment methods typically employed in these environments are reliant upon quantitative measurements as the main identifying feature for talent selection [8]. This study challenges this dominance through a consideration of the benefits and limitations in such a one dimensional approach when used with an adolescent age group, in which relative age effects as well as biases towards the early maturing, physically superior player may be prevalent [9].

At junior level, with rugby league players aged 6-14 years, player development has become increasingly focused on the qualitative development of movements specific to the game $[10,11]$. In contrast, the majority of players within the England Talent Pathway and professional rugby league clubs are commonly assessed using traditional fitness testing batteries (e.g. Nike SPARQ Rugby protocol, [12]). Aligned to this, previous researchers predominantly explored the use of fitness measures including speed, agility, power, strength and aerobic capacity [9,13-15]. This assessment continuum disregards the suggestion that movement development should be viewed from a lifespan perspective and should continue through adolescence alongside physical development [7].

\section{DEVELOPING A DUALISTIC MOVEMENT ASSESSMENT PROTOCOL}

Meylan et al. [8] acknowledged that a one-dimensional approach using physiological and anthropometrical assessment to identify talent, despite being traditional, is misleading and outdated. It has also been suggested that in order to develop high levels of physical literacy a player should develop competence in a variety of movements, applying them in a range of different environments [16]. In order to track development and identify talent accurately, it is important to use assessments of competency appropriate to the age group and the sport. With these developmental perspectives in mind, the RFL proposed the use of a movement assessment protocol (MAP) consisting of two testing batteries to be used within the EtP programme; a qualitative movement assessment (QMA) and a fitness assessment.

The QMA measures specialised skills, typically found in the game of rugby league, that involve the combination and refinement of three categories of movement (locomotor, 
manipulative and stability) [7]. For example, the hop, stick and grip assesses all three movement categories typically encountered by a player being tackled (Table 1).

The physical assessment draws from a range of previously validated measures established to assess physical fitness in relation to speed, agility and whole body power [17]. The physical assessment was also designed in recognition of the recommendation that physical conditioning of pre-pubescent players should be avoided, with an alternative focus on the development of motor skills deemed more appropriate $[18,19]$. Due to the nature and duration of the assessment it has also been advised that pre-pubescent players' performance could be metabolically limited [20]. Based on these perspectives, the use of assessments of anaerobic and aerobic capacity is questionable. This is not the case with respect to the physical assessments that were selected for use within the MAP, with maturation having both positive and negative effects on speed, agility, strength and power tests [21-26].

\section{RELATIVE AGE EFFECTS, MATURATION AND ANTHROPOMETRICS}

Youth rugby is divided into annual age groups to ensure fair and safe competition takes place between players. However, inequalities favouring the player born earlier in the year have been evident with regards to participation in the game and subsequent selection to representative squads $[13,27]$. A relatively older player can often measure higher anthropometrically than their younger counterparts [13]. This may then lead to bias in their selection over their younger peers due to the coaches' perceptions of positive links between greater size and increased performance within the game [27]. However, other evidence suggests relatively high anthropometric measurements do not always lead to increased physiological performance [13].

These inequalities in participation and selection have been termed relative age effects (RAE) and have been attributed to individual differences in maturational status [28]. Evidence of the RAE has been found in a range of sports [27], including rugby league [13]. This is not the case in sports where movement skills are suggested to play a more important role than physiological fitness $[29,30]$. These findings strengthen the need for the evaluation of a dualistic approach to reduce the risk of RAE in a talent identification environment.

During maturation, whole body development occurs leading to increased muscle mass and subsequently strength and power [21]. This again creates inequalities when the early maturing player is more likely to be selected to talent squads and subsequently exposed to higher quality training and coaching [27], particularly when considering the notion that players do not maintain this advantage into adulthood [31]. Therefore, this study explored the relationship between the results of fitness and movement assessments, relative age, maturation and anthropometric characteristics of Under 14 junior Rugby League players, within the context of a talent development intervention.

\section{METHOD}

\section{PARTICIPANTS}

Eighty four players (age $M=14.14$ years, $S D=0.29$, range 13.6-14.6 years) from rugby league community clubs were invited to attend one of three development days held locally to them at different regions within the UK.

Institutional ethical approval was granted for the study and parental consent and player assent was sought and provided prior to the commencement of data capture.

\section{ANTHROPOMETRICS}

Anthropometric measurements were taken for height $(M=169.9 \mathrm{~cm}, S D=7.9$, range 147- 
$187 \mathrm{~cm})$, sitting height $(M=83.5 \mathrm{CM}, S D=5.1$, range $67-98 \mathrm{~cm})$ and body mass $(M=65.6$ $\mathrm{kg}, S D=13.6 \mathrm{~kg}$, range $39-109 \mathrm{~kg}$ ). Height and sitting height were recorded to the nearest $0.1 \mathrm{~cm}$ using a Seca Alpha stand. Body mass was measured using calibrated Seca Alpha (mode 770) scales.

\section{RELATIVE AGE}

The date of birth for each player was recorded and categorized into one of 4 birth quartiles $(\mathrm{Q})$, as previously documented [13,27] $(\mathrm{Q} 1$ = September-November, Q2 = DecemberFebruary, Q3 = March-May, Q4 = June-August) based on the selection start date of 1st September used for creating chronological annual-age groups in UK rugby league.

\section{MATURATION}

Age at peak height velocity (PHV) is the most common measurement of maturational status and was calculated using an age at PHV prediction equation [32]. This method used a genderspecific multiple regression equation including height, sitting height, leg length, body mass, chronological age, and their interactions to estimate age at PHV [33]. Years from PHV (YPHV) was then calculated by subtracting age at PHV from chronological age. Following the estimation of YPHV, participants were categorised into one of three groups (i.e., Earlier, Average, Later) based on their YPHV [9]. The boundaries between the three groups were established by subtracting and adding 0.5 to the mean YPHV $(0.1+/-0.78)$. Players were categorised into later $(-0.4$ years or below, $n=21)$, average (between -0.39 and 0.59 years, $\mathrm{n}=42)$ and earlier maturing $(0.6$ years and above, $\mathrm{n}=21)$ groups. This resulted in a minimum of 1 year difference in maturation between the later and earlier-maturing groups.

\section{MOVEMENT ASSESSMENT PROTOCOL}

Participants were advised to wear appropriate clothing and footwear for testing. All participants were provided with a standardised warm up approximately 15 minutes in duration. The warm-up included a range of dynamic movements followed by moderate to high intensity sprints. Participants were split into groups and performed the tests on a rotation system. Tests were performed on a range of surfaces both indoor and outdoor and were all completed within a two hour period on the same day.

The MAP involved a combination of fitness and qualitative movement assessments, as follows.

\section{Fitness Tests}

Sprint speed was measured over 20 metres using Brower Timing Systems (IR Emit, Draper, Utah, USA). Players began in their own time from a staggered standing start and times were recorded to the nearest $0.01 \mathrm{~s}$. A previous study using the same test and equipment has displayed high reliability (intraclass correlation coefficients were $r=0.852, p<0.001$ ) [14]. Multidirectional speed was assessed using the zigzag agility test. Participants accelerated and performed four changes in direction before finishing with a linear sprint back through the timing systems. Again Brower Timing Systems were used recording times to the nearest $0.01 \mathrm{~s}$.

A countermovement jump with hands kept on hips was performed using a Just Jump Mat (Probotics, Alabama, USA) and recorded to the nearest centimetre. This has been recommended as one of the most valid and reliable measures of lower body power compared with other jump tests [34]. Upper body power was assessed through a maximal horizontal chest throw of a $2 \mathrm{~kg}$ medicine ball (Max Grip, Guangdong, China). 
The players completed the fitness tests in a rotation, from different starting points with the following tests completed in the morning: (i) Vertical Jump, (ii) Medicine ball throw, (iii) Power pass and (iv) Turn off either foot.

\section{Qualitative Movement Assessment}

The QMA assessed seven specialised movements that related to those performed within rugby league and the protocol focused on three key classifications of movement: Stability, locomotion and manipulation [35]. As all participants were around 14 years of age, specialized movement skills were assessed. Specialized movement skills are task specific in that they are "mature fundamental movement patterns that have been refined and combined to form sport skills' [35, p.313]. Due to the limitation in existing motor proficiency assessments for the specific age group of participants, a number of protocols were drawn from and adapted to establish the QMA [36,37]. Each movement was designed to replicate a key movement within the sport of Rugby League and all seven movements were identified and rationalised by a technical group consisting of experts from the RFL. The premise for the inclusion of these assessments was also based on previous studies that had identified movement requirements and current deficiencies of players in rugby league, in relation to the same movement classifications [37]. This is illustrated in Table 1.

Each movement was split up into five components, which assessed an athlete's competency in that specific movement on a scale of 1-5. These components differed across the movements being assessed with some assessing the ability to maintain a given posture (e.g. Superman) and others established to assess different phases of a movement, in a dynamic sense (e.g. hop, stick and grip) (See Table 1). These five values could be added together providing an overall score out of 25 for each movement. Using the body weight squat as an example, the five components were depth, heel movement, back angle, lower limb alignment and weight distribution. If an athlete could not reach a position in which their thighs were parallel with the floor they would receive a score lower than 5. Criteria were provided on each assessment scoring rubric as a guideline for assessors to score, and to minimise the risk of subjective coaches' scoring. Two assessors assessed each QMA movement with a reliable level of agreement demonstrated between the two test raters, which resulted in Kappa values $>0.80$ for each of the assessments. The QMA was conducted in the afternoon with groups starting off at different positions and then rotating in the following order: (i) 20m sprint, (ii) Zigzag agility, (iii) Hop, stick and grip, (iv) Superman, (v) Squat.

\section{DATA ANALYSIS}

Data was analysed using SPSS Statistics Software Version 20. Means and standard deviations were calculated for all variables. In order to investigate differences between relative age quartiles and maturational groups, a one-way analysis of variance (ANOVA) was used. Post-hoc Bonferroni tests were also performed to highlight which individual groups displayed significant differences. To investigate the relationships between height and body mass with MAP variables, a Pearson product correlation coefficient was conducted. Statistical significance was set at $\mathrm{p}<0.05$ in all analyses.

\section{RESULTS}

The anthropometric, fitness and QMA scores by relative age quartile (i.e., Q1, Q2, Q3 and Q4) can be observed in Table 2. A significant effect of relative age for chronological age, YPHV, height, sitting height and body mass was noted, the relatively older (Q1) players more mature, taller and heavier than relatively younger (Q4) players. No significant differences were identified for any fitness or QMA measure. 


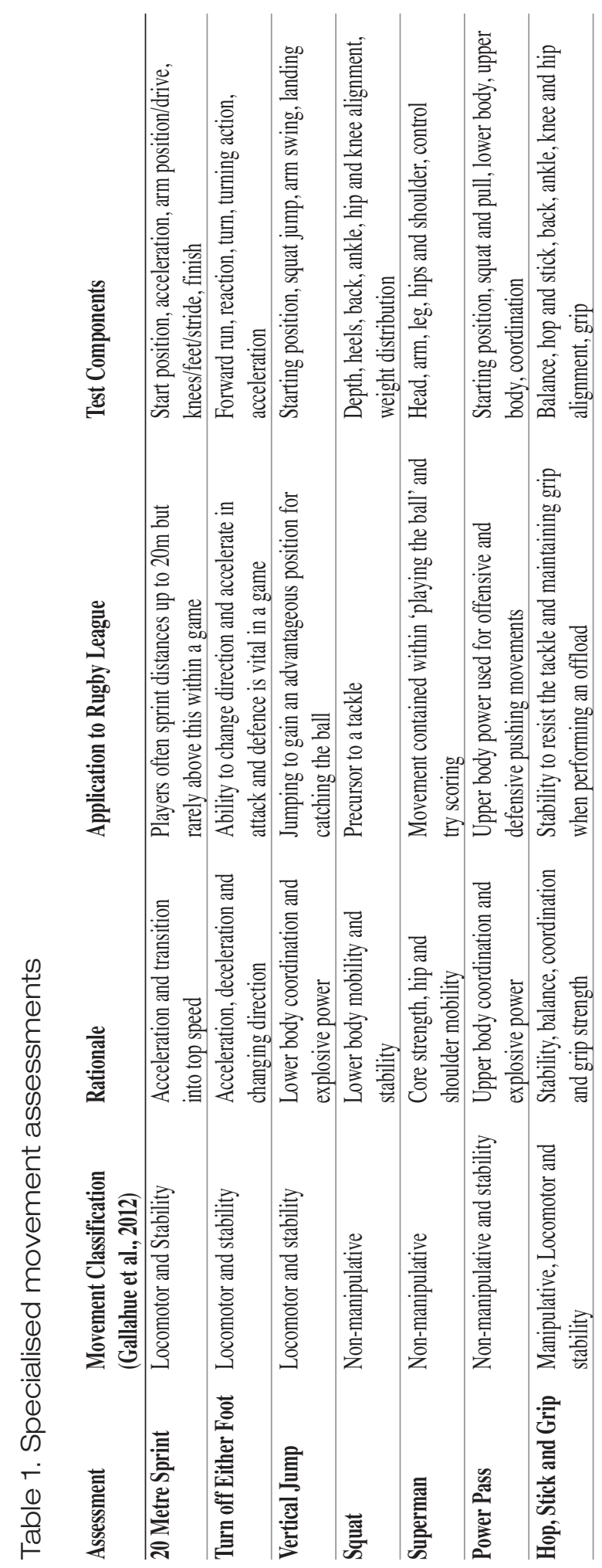


Table 2. Comparisons between relative age quartile and anthropometric characteristics, fitness and QMA scores

\begin{tabular}{|c|c|c|c|c|c|c|c|c|c|c|c|}
\hline \multirow{2}{*}{ Characteristics } & \multicolumn{2}{|c|}{ Quartile 1} & \multicolumn{2}{|c|}{ Quartile 2} & \multicolumn{2}{|c|}{ Quartile 3} & \multicolumn{2}{|c|}{ Quartile 4} & \multicolumn{3}{|c|}{ ANOVA } \\
\hline & $\mathrm{N}$ & $\mathrm{M}(\mathrm{SD})$ & $\mathrm{N}$ & $\mathrm{M}(\mathrm{SD})$ & $\mathrm{N}$ & $\mathrm{M}(\mathrm{SD})$ & $\mathrm{N}$ & $\mathrm{M}(\mathrm{SD})$ & $F$ & p & Post-hoc \\
\hline Chronological Age (years) & 23 & $14.51 \pm 0.07$ & 18 & $14.23 \pm 0.08$ & 28 & $14.00 \pm 0.07$ & 15 & $13.71 \pm 0.09$ & 367.58 & $<0.001$ & $1>2>3>4$ \\
\hline Years from PHV & 23 & $0.57 \pm 0.60$ & 18 & $0.08 \pm 0.59$ & 28 & $0.14 \pm 0.69$ & 15 & $-0.69 \pm 0.84$ & 10.55 & $<0.001$ & $1,3>4$ \\
\hline Height $(\mathrm{cm})$ & 23 & $172.96 \pm 7.44$ & 18 & $169.44 \pm 6.53$ & 28 & $170.18 \pm 8.20$ & 15 & $165.00 \pm 8.01$ & 3.33 & 0.02 & $1>4$ \\
\hline Sitting Height (cm) & 23 & $85.35 \pm 3.88$ & 18 & $83.44 \pm 4.26$ & 28 & $84.25 \pm 5.03$ & 15 & $79.33 \pm 5.95$ & 5.20 & 0.002 & $1,3>4$ \\
\hline Body Mass (kg) & 23 & $72.49 \pm 15.40$ & 18 & $62.24 \pm 8.16$ & 28 & $65.87 \pm 12.02$ & 15 & $58.71 \pm 14.77$ & 4.03 & 0.01 & $1>4$ \\
\hline \multicolumn{12}{|l|}{ Fitness Tests } \\
\hline Vertical Jump (cm) & 23 & $47.58 \pm 8.40$ & 18 & $49.20 \pm 6.21$ & 27 & $46.50 \pm 7.48$ & 15 & $45.17 \pm 5.11$ & 0.99 & 0.40 & \\
\hline Medicine ball throw (m) & 23 & $5.01 \pm 0.65$ & 18 & $5.00 \pm 0.63$ & 27 & $4.84 \pm 0.62$ & 15 & $4.72 \pm 0.61$ & 0.87 & 0.46 & \\
\hline $20 \mathrm{~m}$ Sprint $(\mathrm{s})$ & 23 & $3.52 \pm 0.23$ & 18 & $3.53 \pm 0.22$ & 26 & $3.57 \pm 0.23$ & 15 & $3.65 \pm 0.16$ & 1.20 & 0.32 & \\
\hline Zigzag Agility (s) & 23 & $11.98 \pm 1.00$ & 18 & $11.75 \pm 0.77$ & 26 & $12.40 \pm 0.72$ & 15 & $12.28 \pm 0.82$ & 2.57 & 0.06 & \\
\hline \multicolumn{12}{|l|}{$Q M A$} \\
\hline Vertical Jump Score & 23 & $14.22 \pm 2.70$ & 18 & $14.28 \pm 2.87$ & 27 & $13.59 \pm 2.50$ & 15 & $13.87 \pm 2.07$ & 0.36 & 0.78 & \\
\hline Power Pass Score & 23 & $13.30 \pm 2.18$ & 18 & $13.61 \pm 2.22$ & 27 & $13.15 \pm 2.48$ & 15 & $13.33 \pm 2.06$ & 0.15 & 0.93 & \\
\hline Sprint Score & 23 & $13.61 \pm 2.19$ & 18 & $13.78 \pm 1.83$ & 26 & $14.08 \pm 1.38$ & 15 & $13.93 \pm 1.75$ & 0.30 & 0.83 & \\
\hline Turn off Either Foot Score & 20 & $12.70 \pm 3.28$ & 17 & $13.76 \pm 4.10$ & 25 & $14.76 \pm 3.57$ & 13 & $13.92 \pm 3.62$ & 1.20 & 0.32 & \\
\hline Superman Score & 18 & $16.28 \pm 1.23$ & 17 & $15.47 \pm 2.07$ & 25 & $15.20 \pm 2.24$ & 13 & $15.69 \pm 2.66$ & 0.97 & 0.41 & \\
\hline Squat Score & 17 & $17.18 \pm 3.36$ & 16 & $17.75 \pm 4.25$ & 21 & $17.52 \pm 3.33$ & 11 & $16.82 \pm 3.06$ & 0.18 & 0.91 & \\
\hline Hop, Stick, Grip Score & 20 & $15.05 \pm 4.14$ & 18 & $15.33 \pm 4.40$ & 24 & $15.58 \pm 1.87$ & 10 & $14.73 \pm 1.83$ & 0.23 & 0.87 & \\
\hline
\end{tabular}

Table 3. Comparisons between maturation group and anthropometric characteristics, fitness and QMA scores

$\begin{array}{llll}\text { Characteristics } & \text { Earlier Maturing } & \text { Average Maturing } & \text { Later Maturing }\end{array}$
(E)
(A)
(L)

\begin{tabular}{|c|c|c|c|c|c|c|c|c|c|}
\hline & $\mathbf{N}$ & $\mathrm{M}(\mathrm{SD})$ & $\mathrm{N}$ & $\mathrm{M}(\mathrm{SD})$ & $\mathbf{N}$ & $\mathrm{M}(\mathrm{SD})$ & $\mathrm{F}$ & $p$ & Post-hoc \\
\hline Chronological Age (Years) & 21 & $14.28 \pm 0.28$ & 42 & $14.16 \pm 0.27$ & 21 & $13.94 \pm 0.27$ & 8.47 & $<0.001$ & $E, A>L$ \\
\hline Years from PHV & 21 & $1.00 \pm 0.40$ & 42 & $0.18 \pm 0.24$ & 21 & $-0.96 \pm 0.47$ & 163.65 & $<0.001$ & $E>A>L$ \\
\hline Height $(\mathrm{cm})$ & 21 & $178.33 \pm 4.91$ & 42 & $170.24 \pm 4.62$ & 21 & $160.62 \pm 5.35$ & 69.35 & $<0.001$ & $E>A>L$ \\
\hline Sitting Height $(\mathrm{cm})$ & 21 & $89.14 \pm 2.82$ & 42 & $84.07 \pm 1.99$ & 21 & $76.71 \pm 3.18$ & 127.57 & $<0.001$ & $E>A>L$ \\
\hline Body Mass (kg) & 21 & $78.36 \pm 12.05$ & 42 & $65.51 \pm 10.94$ & 21 & $53.12 \pm 6.40$ & 31.39 & $<0.001$ & $E>A>L$ \\
\hline \multicolumn{10}{|l|}{ Fitness Tests } \\
\hline Vertical Jump (cm) & 21 & $46.62 \pm 5.79$ & 41 & $48.51 \pm 8.04$ & 21 & $45.00 \pm 6.04$ & 1.79 & 0.17 & \\
\hline Medicine Ball Throw (m) & 21 & $5.39 \pm 0.46$ & 41 & $4.90 \pm 0.48$ & 21 & $4.42 \pm 0.68$ & 17.22 & $<0.001$ & $E>A>L$ \\
\hline 20m Sprint (s) & 21 & $3.48 \pm 0.14$ & 40 & $3.56 \pm 0.25$ & 21 & $3.65 \pm 0.19$ & 3.29 & 0.04 & $\mathrm{E}<\mathrm{L}$ \\
\hline Zigzag Agility (s) & 21 & $11.92 \pm 0.83$ & 40 & $12.17 \pm 0.86$ & 21 & $12.22 \pm 0.90$ & 0.77 & 0.47 & \\
\hline \multicolumn{10}{|l|}{$Q M A$} \\
\hline Vertical Jump Score & 21 & $14.48 \pm 2.52$ & 41 & $13.98 \pm 2.71$ & 21 & $13.43 \pm 2.20$ & 0.89 & 0.41 & \\
\hline Power Pass Score & 21 & $13.57 \pm 2.14$ & 41 & $13.88 \pm 2.18$ & 21 & $12.00 \pm 1.98$ & 5.64 & 0.005 & $A>L$ \\
\hline Sprint Score & 21 & $13.38 \pm 2.44$ & 40 & $13.90 \pm 1.48$ & 21 & $14.24 \pm 1.48$ & 1.25 & 0.29 & \\
\hline Turn off Either Foot Score & 19 & $13.21 \pm 3.98$ & 38 & $14.34 \pm 3.44$ & 18 & $13.44 \pm 3.71$ & 0.75 & 0.48 & \\
\hline Superman Score & 19 & $16.05 \pm 1.90$ & 36 & $15.69 \pm 2.04$ & 18 & $15.00 \pm 2.30$ & 1.24 & 0.30 & \\
\hline Squat Score & 19 & $16.47 \pm 3.37$ & 31 & $17.90 \pm 3.82$ & 15 & $17.40 \pm 2.77$ & 1.00 & 0.38 & \\
\hline Hop, Stick, Grip Score & 20 & $15.80 \pm 2.19$ & 36 & $15.64 \pm 3.48$ & 21 & $13.95 \pm 3.38$ & 2.33 & 0.10 & \\
\hline
\end{tabular}


Anthropometric, fitness and QMA scores by maturation group (i.e., Earlier, Average, Later) are detailed in Table 3. Significant effects for maturation group for age at PHV, YPHV, height, sitting height, body mass, medicine ball throw, 20m sprint and power pass QMA was noted. The earlier maturing players were older, taller (11.0\%) and heavier $(47.4 \%)$ than later maturing players. For the medicine ball throw, Earlier outperformed Average who outperformed Later maturers (22.7\% between Early and Later) and for $20 \mathrm{~m}$ sprint the earlier maturing group outperformed the later maturing group (4.7\%). For power pass QMA score the average maturers outperformed the later maturers (15.7\%). No significant differences were identified for any other fitness or QMA measure.

The relationship between height and body mass with fitness and QMA performance can be observed in Table 4. Height was related to $20 \mathrm{~m}$ sprint performance $(\mathrm{r}=-0.31)$, medicine ball throw $(\mathrm{r}=0.53)$ and power pass QMA $(\mathrm{r}=0.32)$ ). Body mass was positively related to medicine ball throw $(\mathrm{r}=0.50)$ and power pass QMA $(\mathrm{r}=0.22)$, a greater body mass resulting in improved performance. However, for vertical jump $(\mathrm{r}=-0.24)$, sprint QMA $(\mathrm{r}=-0.29)$ and turn off either foot QMA ( $\mathrm{r}=-0.26)$ the relationship was negative suggesting greater body mass is associated with worse performance in these measures.

Table 4. Correlations ( $r$ ) and 95\% confidence intervals $(\mathrm{Cl})$ between height and body mass with fitness and QMA Scores

\begin{tabular}{cccc} 
& Variable & Height & Body Mass \\
\hline Fitness & & & \\
\hline & 20m Sprint & $-0.31(-0.51--0.13)^{* *}$ & $0.12(-0.11-0.25)$ \\
\hline & Vertical Jump & $0.20(0.01-0.50)$ & $-0.24(-0.37-0.14)^{*}$ \\
\hline Zigzag Agility & $-0.18(-0.39-0.08)$ & $0.09(-0.12-0.32)$ \\
\hline QMA & $0.53(0.42-0.70)^{* * *}$ & $0.50(0.37-0.74)^{* * *}$ \\
\hline & & \\
\hline & & $0.05(-0.08-0.37)$ \\
\hline & Verticine Ball Throw & $0.20(-0.05-0.45)$ & $0.22(-0.06-0.45)^{*}$ \\
\hline Power Pass QMA & $0.32(0.08-0.54)^{* *}$ & $-0.29(-0.52-0.03)^{* *}$ \\
\hline Sprint QMA & $-0.17(-0.51-0.05)$ & $-0.26(-0.51--0.00)^{*}$ \\
\hline Turn Off Either Foot QMA & $-0.07(-0.39-0.18)$ & $0.16(-0.06-0.48)$ \\
\hline Superman QMA & $0.11(-0.13-0.41)$ & $-0.20(-0.45-0.02)$ \\
\hline Squat QMA & $-0.09(-0.31-0.08)$ & $-0.02(-0.25-0.31)$ \\
\hline Hop, Stick and Grip QMA & $0.08(-0.02-0.46)$ &
\end{tabular}

Significant correlations between variables: ${ }^{*} \mathrm{p}<0.05 ; * * \mathrm{p}<0.01 ; * * * \mathrm{p}<0.001$

\section{DISCUSSION}

The aim of this study was to explore the relationships between the results of fitness and movement assessments, relative age, maturation and anthropometric characteristics of Under 14 junior Rugby League players, within the context of a talent development intervention.

\section{RELATIVE AGE}

This study found significant differences between relative age quartiles for body mass, which is consistent with previous research findings [14]. In contrast to Till et al. [14], this study found players born in the first quartile of the year were significantly heavier than those born in the fourth quartile. Significant differences were evident between relative age groups for height and sitting height in this study, which contradicts previous research [14]. Players born 
in Q1 were significantly taller, heavier and more mature, compared to Q4 players. Till et al. [14] showed age at PHV occurred significantly earlier in Quartile 4 players (13.39 \pm 0.59 years) compared to Quartile 1 players (13.65 \pm 0.57 years). Previous research [14] was conducted in a high performance sample of junior rugby league players whereas the current community sample had no identification or selection processes. This suggests relatively younger players selected into a development squad must have anthropometric characteristics matching relatively older players, which does not seem evident in the community game where no selection or identification processes take place.

A key finding from this sample is that, despite displaying greater anthropometric characteristics, relatively older players did not perform significantly better on any fitness assessment than the relatively younger players. This finding supports previous research in rugby league $[9,14]$ and football $[38]$ that differences in fitness performance are negligible when compared across chronological age quartiles. Although this is surprising based on the general relationships between age and fitness performance, it suggests that talent identification and selection processes make selections based on advanced anthropometric characteristics rather than physical performance. These findings emphasise the issue in a talent identification environment when the relatively older player is more likely to be selected $[13,28]$. Greater anthropometric characteristics are desirable to coaches, who perceive them as beneficial to physiological performance within the sport [14,29]. The findings from this study suggest that players born earlier in the year may appear physically superior but in fact, players within an annual age group were homogenous with regard to fitness performance, regardless of chronological age. This is consistent with previous findings in rugby league $[9,14]$ and soccer $[38,39]$.

This study found no significant differences between chronological age quartiles in qualitative movement performance. This implies that a player born later in the selection year has no disadvantage when assessed qualitatively compared to players born earlier in the year. This view is shared by previous research that suggests relative age effects are not present when a more qualitative approach is used [29,30]. These findings promote the idea of a qualitative approach to movement assessment and its potential to reduce the occurrence of relative age effects. Cobley et al. [27] share a similar view suggesting that while accounting for relative age effects, coaches need to integrate a movement based approach to talent selection which reduces the dependence upon physiological fitness attributes.

\section{MATURATION}

Findings demonstrated significant differences between maturational groups (i.e., Earlier, Average, Later) for anthropometric measurements. The results were tiered as earlier maturing players were significantly taller and heavier than average maturing players, with later maturing players the smallest and lightest. This demonstrates that advanced maturation provides increased anthropometric characteristics, supporting previous research findings $[9,33,40]$.

One key finding within this sample was that maturation did influence performance in some of the fitness assessments (i.e., medicine ball throw and $20 \mathrm{~m}$ sprint). A tiered trend was evident as earlier maturers outperformed average maturers, who in turn outperformed later maturers, in the medicine ball throw assessment. This finding is consistent with that of Till et al. [9] and implies those who mature early have an enhanced upper body power due to the relationships between maturation and power output. This could be explained by increases in the upper body strength and power development that are suggested to occur at a greater rate compared to development of other parts of the body during adolescence [21,22]. The 
recommendation that maturation is strongly related to upper body power has been consistently found $[9,15,41]$. As a result, it is questionable as to whether the power pass test is an appropriate assessment of ability when used with under 14 Rugby League players, as early maturing players are advantaged.

Another interesting finding was that earlier maturing players significantly outperformed later maturing players in the 20 metre sprint. This has been reported in early research [41], but was not found to be the case in more recent research [9]. The bias towards early maturers is plausible due to the rapid increases in strength and hormone-dependant hypertrophy of fast-twitch muscle fibres that occurs between 13-15 years in boys [21,42]. This raises questions over the appropriateness of sprint testing in under 14 players. However, it has been suggested that an early maturing player's sprint ability may be hindered as well as facilitated during adolescence as a result of their early maturation. Stafford [43] suggests that when players reach PHV they experience rapid growth of limbs and increased strength, allowing them to cover more ground through an increased stride length and at a quicker rate. However, players may also experience changes in their centre of mass, which can negatively affect stability and acceleration [43]. These findings suggest that the assessment of a player's sprinting competence needs to be measured alongside sprint speed while considering maturation status.

The significant differences observed between maturity groups for medicine ball power pass and $20 \mathrm{~m}$ sprint demonstrates that fitness performance may be more related to maturity than chronological age, due to no differences observed between relative age quartiles. This suggests that coaches should assess fitness performance in relation to both age and maturation, using non-invasive methods, similar to those used in the current study.

Significant differences were apparent between average and later maturing players in the power pass QMA. A possible explanation may be that the later maturing player may have a reduced upper body development compared to a more mature player, as this is one of the earliest developments during adolescence $[21,22]$. Further, the medicine ball may be too heavy and difficult to manipulate for later maturing players and therefore affect the ability to execute the action effectively. The later maturing player may also struggle to perform the assessment physically due to lesser ability to generate strength and power output [21]. To minimise the bias against the later maturing player, the requirement of measuring upper body strength and power may need to be removed.

\section{RELATIONSHIP BETWEEN HEIGHT AND BODY MASS WITH THE MAP}

Body mass was negatively related to vertical jump performance, which has been found in previous research [14] and could be attributed to a reduced power to body mass ratio [17]. This emphasizes the point that, despite displaying superior body mass, the earlier maturing player does not necessarily possess an increased vertical jump performance.

Increased sprint performance was moderately associated with height, which could be attributed to an increased muscle development and stride length [43]. Body mass displayed no significant negative relationship to sprint performance in contrast to Till et al. [14] who found that sprint performance decreased alongside increases in body mass. No correlations were found between height and body mass with agility performance, although this assessment was different to that used in previous studies $[14,17]$.

Within this sample, medicine ball throw performance had a strong and positive relationship with height and body mass. Once more, this has been previously evident [14] and could be attributed to increased upper body development during maturation $[21,22]$ and an increased lever length, which leads to an ability to generate more force [44]. This 
reinforces the suggestion that this assessment creates a bias towards the earlier maturing player. To conclude, anthropometrics may have a positive influence on performance but likewise they can have a negative one.

Body mass was negatively related to sprint and turn off either foot QMA performance within this study. As previously discussed a heavier player with a reduced power: body mass ratio [17] may have a reduced ability to change their centre of mass quickly when accelerating and changing direction quickly [44]. With the two assessments heavily based on speed of movement, a reduced ability to display this may lead to the perception of a lower movement quality from assessors, hence the correlation. Previous research has also found strong inverse relationships between body fat and locomotor movement skills [45]. In contrast, body mass and height correlated positively to power pass QMA. This could be attributed to a greater rate of upper body development and subsequent easier ability to manipulate the medicine ball $[21,22]$

\section{LIMITATIONS}

This study is not without limitations. Firstly, body fat percentage was not assessed and the influence of fat mass upon performance could not be gauged [14]. The use of the age at PHV prediction equation as a measure of maturation has not been correlated with other maturity indicators (i.e., skeletal age, secondary sexual characteristics) within athletes, therefore questioning its reliability. However, while accepting potential error, an assessment of maturation remains beneficial, as it is a simple non-intrusive way of predicting maturation as applied in other research [9,33].

The QMA also had limitations as it lacked in manipulative movement assessment. The qualitative assessment used 7 assessments although more may be required to assess key manipulative movements such as passing and catching. However, standardisation in natural settings can be difficult due to the impact of external influences; for example, if catches were received from a handfed pass there is a reliance on the quality and consistency of the feed. These issues need to be considered when the QMA protocol is used in future situations. Finally, all participants used within this study had experience of playing rugby league. However, the Embed the Pathway project is aimed at those who may not have played the sport before or have withdrawn from the sport at an earlier age. Different findings may have been present if a fully representative target population of the project was used within this study.

\section{CONCLUSION}

Relatively older players demonstrated greater anthropometrical measurements, but did not perform better in the MAP. Coaches need to be educated on the potential effects of relative age within annual-age groups on size and performance. Findings demonstrate that maturation also has a significant impact on anthropometric measures along with upper body power and sprint speed. These findings question the use of upper body power and sprint speed assessments within talent identification programs by annual-age category. Instead, practitioners should consider fitness assessments in relation to maturational status when assessing an individual's performance. Further, anthropometric characteristics have positive and negative effects on performance, of which coaches should be aware to avoid selection of players based on size, which may actually be detrimental to fitness and movement ability (e.g., sprint speed and agility). Future research could investigate the effect of aerobic and anaerobic performance between maturational groups to explore the appropriateness of such assessments within the context of talent development. Additionally, research investigating 
movement development and assessment in under 14 players, whether it be quantitative or qualitative, should carefully consider chronological age, maturational status and anthropometrics in relation to performance. If Movement Assessment Protocols are to be used for the purpose of talent identification they should not consist of assessments that allow for relative age and maturational biases and should be developmentally appropriate for the target population [27].

\section{ACKNOWLEDGEMENTS}

The Rugby Football League funded this research. Thank you to the RFL and all of the participants in the study for allowing us to develop an understanding of this crucial area of work.

\section{REFERENCES}

1. Rugby Football League, Available Online at: http://www.therfl.co.uk. [Accessed 21 ${ }^{\text {st }}$ October, 2013].

2. Malina, R., Eisenmann, J., Cumming, S., Ribeiro, B. and Aroso, J., Maturity-Associated Variation in the Growth and Functional Capacities of Youth Football (Soccer) Players 13-15 years, European Journal of Applied Physiology, 2004, 91, 555-562.

3. Rugby Football League, Available Online at: http://www.therfl.co.uk. [Accessed 10th September 2012].

4. Malina, R., Bouchard, C. and Bar-Or, O., Growth, Maturation, and Physical Activity, Human Kinetics, $2^{\text {nd }}$ edn, Human Kinetics, Champaign, IL, 2004.

5. Gabbett, T., Physiological Characteristics of Junior and Senior Rugby League Players, British Journal of Sports Medicine, 2002, 36, 334-339.

6. Gabbett, T. and Herzig, P., Physiological Characteristics of Junior Elite and Sub-Elite Rugby League Players, Strength and Conditioning Coaching, 2004, 12, 19-24.

7. Gallahue, D. and Ozmun, J., Understanding Motor Development: Infant, Children, Adolescents, Adults, $6^{\text {th }}$ edn., McGraw-Hill, New York, 2006.

8. Meylan, C., Cronin, J., Oliver, J. and Hughes, M., Talent Identification in Soccer: The Role of Maturity Status on Physical, Physiological and Technical Characteristics, International Journal of Sports Science and Coaching, 2010, 5(4), 571-592.

9. Till, K., Cobley, S., O’Hara, J., Cooke, C. and Chapman, C., Considering Maturation Status and Relative Age in the Longitudinal Evaluation of Junior Rugby League Players, Scandinavian Journal of Medicine and Science in Sports, 2013, 16(5), 438-443.

10. Morley, D., A 'Fit for Purpose Strategy' for the Rugby Football League. in, Navin, A., ed., Sports Coaching: a Reference Guide for Students, Coaches and Competitors, Crowood Press, Marlborough, 2011.

11. Morley, D. and Muir, B., Becoming CAYPABLE: Level 1 Coach Qualification Support Resource, Coachwise, Leeds, 2012.

12. Nike., SPARQ Fitness Assessment, Available Online at: http://www.sparqtraining.com/home [Accessed 20 October, 2013].

13. Till, K., Cobley, S., Wattie, N., O'Hara, J., Cooke, C. and Chapman, C., The Prevalence, Influential Factors and Mechanisms of Relative Age Effects in UK Rugby League, Scandinavian Journal of Science and Medicine in Sport, 2010, 20, 320-329.

14. Till, K., Cobley, S., O'Hara, J., Chapman, C. and Cooke, C., Anthropometric, Physiological and Selection Characteristics in High Performance UK Junior Rugby League Players, Talent Development and Excellence, 2010, 2(2), 193-207.

15. Till, K., Cobley, S., O'Hara, J., Brightmore, A., Cooke, C. and Chapman, C., Using Anthropometric and Performance Characteristics to Predict Selection in Junior UK Rugby League Players, Journal of Science and Medicine in Sport, 2011, 14(3), 264-269.

16. Lloyd, M., Colley, R. and Tremblay, M., Advancing the Debate on 'Fitness Testing' for Children: Perhaps We're Riding the Wrong Animal, Pediatric Exercise Science, 2010, 22, 176-182. 
17. Gabbett, T., Applied Physiology of Rugby League, Sports Medicine, 2008, 38, 119-138.

18. Lidor, R., Cote, J. and Hackfort, D., ISSP Position Stand: To Test or Not to Test? The Use of Physical Skill Tests in Talent Detection and in Early Phases of Sport Development, International Journal of Sport and Exercise Psychology, 2009, 9, 131-146.

19. Saltin, B., The Search For and Fostering of the Young Talent, in Hackfort, D., ed., Striving for Excellence, Fitness Information Technology, Morgantown, WV, 2007, 3-19.

20. Bailey, R., Collins, D., Ford, R., MacNamara, A., Toms, M. and Pearce, G., Participant Development in Sport: An Academic Review, Sports Coach UK, Leeds, 2010.

21. Viru, A., Loko, J., Harro, M., Volver, A., Laaneots, L. and Viru, M., Critical Periods in the Development of Performance Capacity During Childhood and Adolescence, European Journal of Physical Education, 1999, 4(1), 75-119.

22. Blanksby, B., Bloomfield, J., Ackland, T., Elliott, B. and Morton, A.R., Athletics, Growth and Development in Children, Harwood Academic Publishers, Camberwell, 1994.

23. Christou, M., Smilios, I., Sotiropoulos, K., Volaklis, K., Pilianidis, T. and Tokmakidis, S., Effects of Resistance Training on the Physical Capacities of Adolescent Soccer Players, Journal of Strength and Conditioning Research, 2006, 20(4), 783-791.

24. Figueiredo, A.J., Goncalves, C.E., Coelho, E.S.M.J. and Malina, R.M., Characteristics of Youth Soccer Players Who Drop Out, Persist or Move Up, Journal of Sports Sciences, 2009, 27(9), 883-891.

25. Saltin, B., The Search For and Fostering of the Young Talent, paper presented at the opening of ASPIRE Academy, Doha, Qatar, 2005.

26. Van Praagh, E., Pediatric Anaerobic Performance, Human Kinetics, Champaign, IL, 1998.

27. Cobley, S., Baker, J., Wattie, N. and McKenna, J., Annual Age-Grouping and Athlete Development: A MetaAnalytical Review of Relative Age Effects in Sport, Sports Medicine, 2009, 39(3), 235-256.

28. Musch, J. and Grondin, S., Unequal Competition as an Impediment to Personal Development: A Review of the Relative Age Effect in Sport, Developmental Review, 2001, 21, 147-167.

29. Baxter-Jones, A.D., Helms, P., Maffulli, N., Baines-Preece, J.C. and Preece, M., Growth and Development of Male Gymnasts, Swimmers, Soccer and Tennis Players: A Longitudinal Study, Annals of Human Biology, 1995, 22(5), 381-394.

30. Van Rossum, J.H., Relative Age Effect Revisited: Findings from the Dance Domain, Perceptual and Motor Skills, 2006, 102(2), 302-308.

31. Beunen, G., Ostyn, M., Simons, J., Renson, R., Claessens, A., VandenEynde, B., Lefevre, J., Vanreusel, B., Malina, R. and van't Hof, M., Development and Tracking in Fitness Components: Leuven Longtudinal Study on Lifestyle, Fitness and Health, International Journal of Sports Medicine, 1997, 18(3), 171-178.

32. Mirwald, R., Baxter-Jones, A., Bailey, D. and Beunen, G., An Assessment of Maturity from Anthropometric Measurements, Medicine and Science in Sports and Exercise, 2002, 34(4), 689-694.

33. Sherar, L., Baxter-Jones, A., Faulkner, R. and Russell K., Do Physical Maturity and Birth Date Predict Talent in Male Youth Ice Hockey Players? Journal of Sports Sciences, 2007, 25, 879-886.

34. Hunter, J. and Marshall, R., Effects of Power and Flexibility Training on Vertical Jump Technique, Medicine and Science in Sports and Exercise, 2002, 34(3), 478-486.

35. Gallahue, D., Ozmun, J. and Goodway, J., Understanding Motor Development in Infants, Children, Adolescents and Adults, 7th edn., MacGraw-Hill, New York, 2012.

36. Bruininks, R. H., Bruininks-Oseretesky Test of Motor Proficiency, American Guidance Service, Circle Pines, MN, 1978 .

37. Burton, A.W. \& Miller, D E., Movement Skill Assessment, Human Kinetics, Champaign, IL, 1998.

38. Carling, C., le Gall, F., Reilly, T. and Williams, A., Do Anthropometric and Fitness Characteristics Vary According to Birth Date Distribution in Elite Youth Academy Soccer Players, Scandinavian Journal of Medicine and Science in Sports, 2009, 19, 3-9.

39. Hirose, N., Relationships Among Birth-Month Distributions, Skeletal Age and Anthropometric Characteristics in Adolescent Elite Soccer Players, Journal of Sports Science, 2009, 27, 1159-1166. 
40. Malina, R., Physical Growth and Biological Maturation of Young Athletes, Exercise and Sport Science Reviews, 1994, 22, 389-433.

41. Lefevre, J., Beunen, G., Steens, G., Claessens, A. and Renson, R., Motor Performance During Adolescence and Age Thirty as Related to Peak Height Velocity, Annals of Human Biology, 1990, 17(5), 423-435.

42. Ford, P., De Ste Croix, M., Lloyd, R., Meyers, R., Moosavi, M., Oliver, J., Till, K. and Williams, C., The Long-Term Athlete Development Model: Physiological Evidence and Application, Journal of Sports Sciences, 2011, 29(4), 389-402.

43. Stafford, I., Coaching Children in Sport, Routledge, Oxford, 2011.

44. McArdle, W., Katch, F. and Katch, V., Exercise Physiology: Energy, Nutrition, and Human Performance, Williams \& Wilkins, $4^{\text {th }}$ edn., Baltimore, 1996.

45. Okely, A., Booth, M. And Chey, T., Relationships Between Body Composition and Fundamental Movement Skills Among Children and Adolescents, Research Quarterly for Exercise and Sport, 2004, 75(3), 238-247. 